\title{
Physicists explain 'gravity-defying' chain trick
}

\section{Leaping beads get a push from the pot.}

\section{Elizabeth Gibney}

15 January 2014

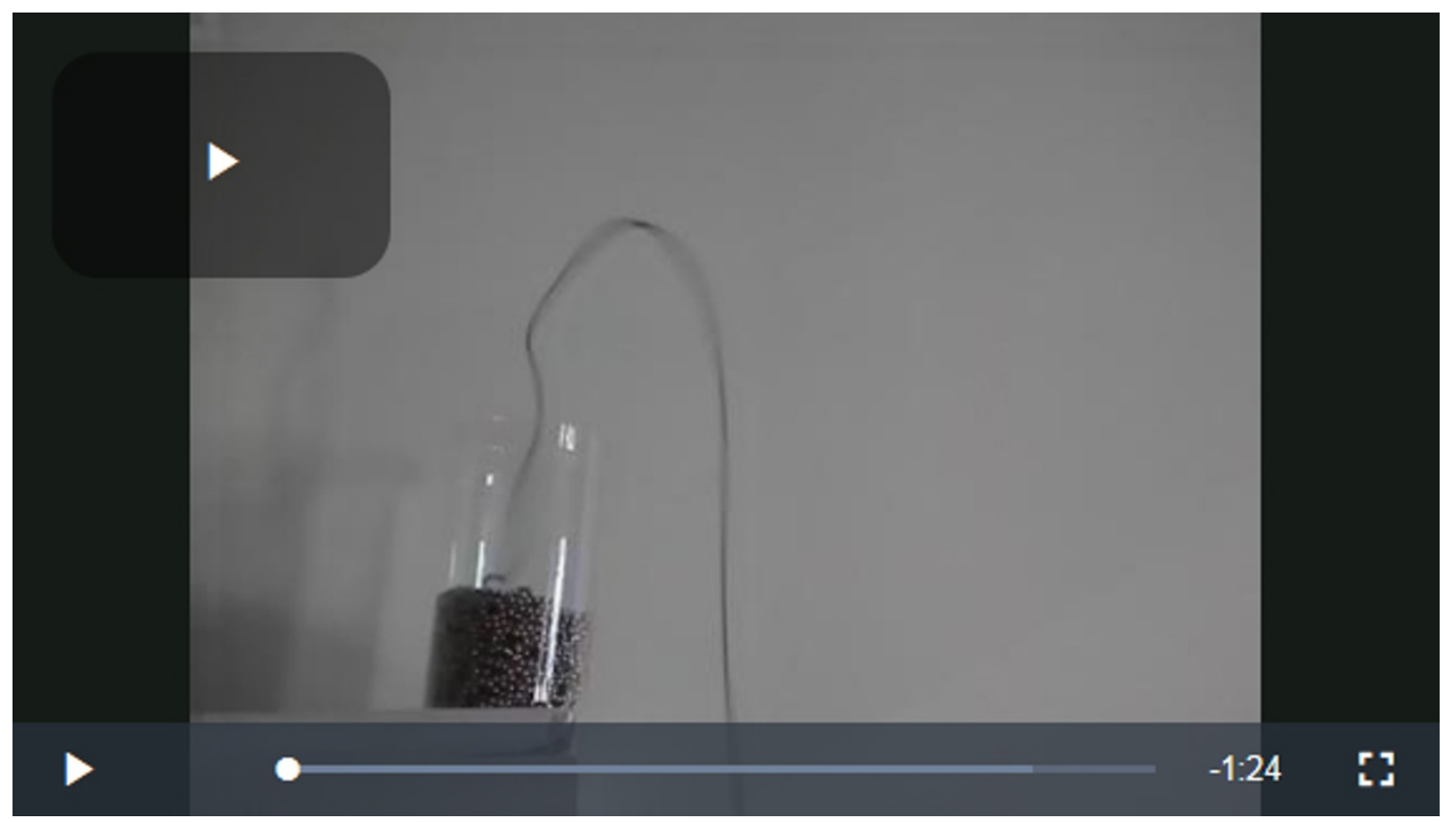

Leaping up out of a jar in an arc before falling to the floor, the fountain-like motion of a chain of beads has puzzled millions around the world with its apparently gravity-defying behaviour. Now physicists think they have an explanation. And it is far from intuitive.

British science presenter Steve Mould, who made the experiment famous with a video that went viral on the Internet, explained the phenomenon as simply one of inertia: the falling chain has downward momentum, causing an upward momentum in beads leaving the pot. This, in turn, makes them leap before gravity can slowly reverse their momentum.

Mould's explanation was clever, but wrong, says physicist John Biggins of the University of Cambridge, UK. If inertia were causing the flowing fountain, the chain would be stationary at the top of the curve, says Biggins, in the same way that a ball tossed into the air is stationary at its highest point. "If that were true, it would mean the chain would pile up in the top region, which we don't see," he adds.

The only way to account for the rise is for the chain to receive a kick from the pot from which it is being pulled, say Biggins and his Cambridge colleague Mark Warner. This challenges not only the explanation given by Mould, but the conventional mathematics of chains.

\section{Rigid discipline}

Rather than a flexible string of isolated beads, the chain is more like a series of short, rigid 'rods', say the authors, who publish their results today in Proceedings of the Royal Society $A^{1}$. In their model, each rod is made up of three beads and two connectors. The size of a rod corresponds to the number of beads it takes to turn a section of chain back on itself by 180 degrees (it takes six).

Picking up a rod from the pot with an upward force on one of its ends causes two things to happen, says Biggins. It makes the rod lift, but it also causes it to rotate. The end that is not picked up pushes downwards, and the pot provides a reaction force, he says. "The far end of the rod under those two motions actually goes down, and therefore pushes down. And that gives rise to this extra kick from the pot which drives the fountain." 
Without the extra kick, the chain would still flow to the floor, but it would run over the side of the beaker without creating the fountain, he says. The duo predicted that experiments using chains made of small, heavy beads separated by string, which get picked up in isolation and do not behave like rods, would produce no fountain. "That was absolutely borne out by the experiment," says Biggins (see video below).

"This is a neat paper," says James Hanna, a mechanician at Virginia Tech, who has tackled similar problems in horizontal chains. He says the explanation is plausible, but may not be the whole story. Although Biggins and Ward suggest that other, sideways forces caused by the configuration of the chain in the pot - might also be at play, ultimately they predict that a chain falling from a given distance produces a steady-height fountain, he says. Real world videos show the chain's behaviour is far from steady, he adds. "I'm not $100 \%$ convinced there is a steady state. I would love to see a really long experiment, with a mile long chain. But that's not really feasible I suppose," he adds.

Biggins and Warner first tackled the fountain problem when looking for physics puzles that could be given to A-level students as a taste of real-world problems. Their solution was inspired by the equally bizarre finding that whereas a pot can provide a pushing effect, a chain falling to the floor also experiences a 'sucking' effect.

Experiments by mechanical engineer Andy Ruina and his colleagues ${ }^{2}$ at Cornell University in Ithaca, New York, showed that when dropping two chains side by side — one onto a table and one into free space — the chain falling to the table falls faster than the one falling in free space. The suck is explained in the same way as the kick. As a section of chain lands, the first end to touch the surface experiences a pushing force, rotating the rod and pulling down the other end, says Biggins. "To my mind, it's completely astounding."

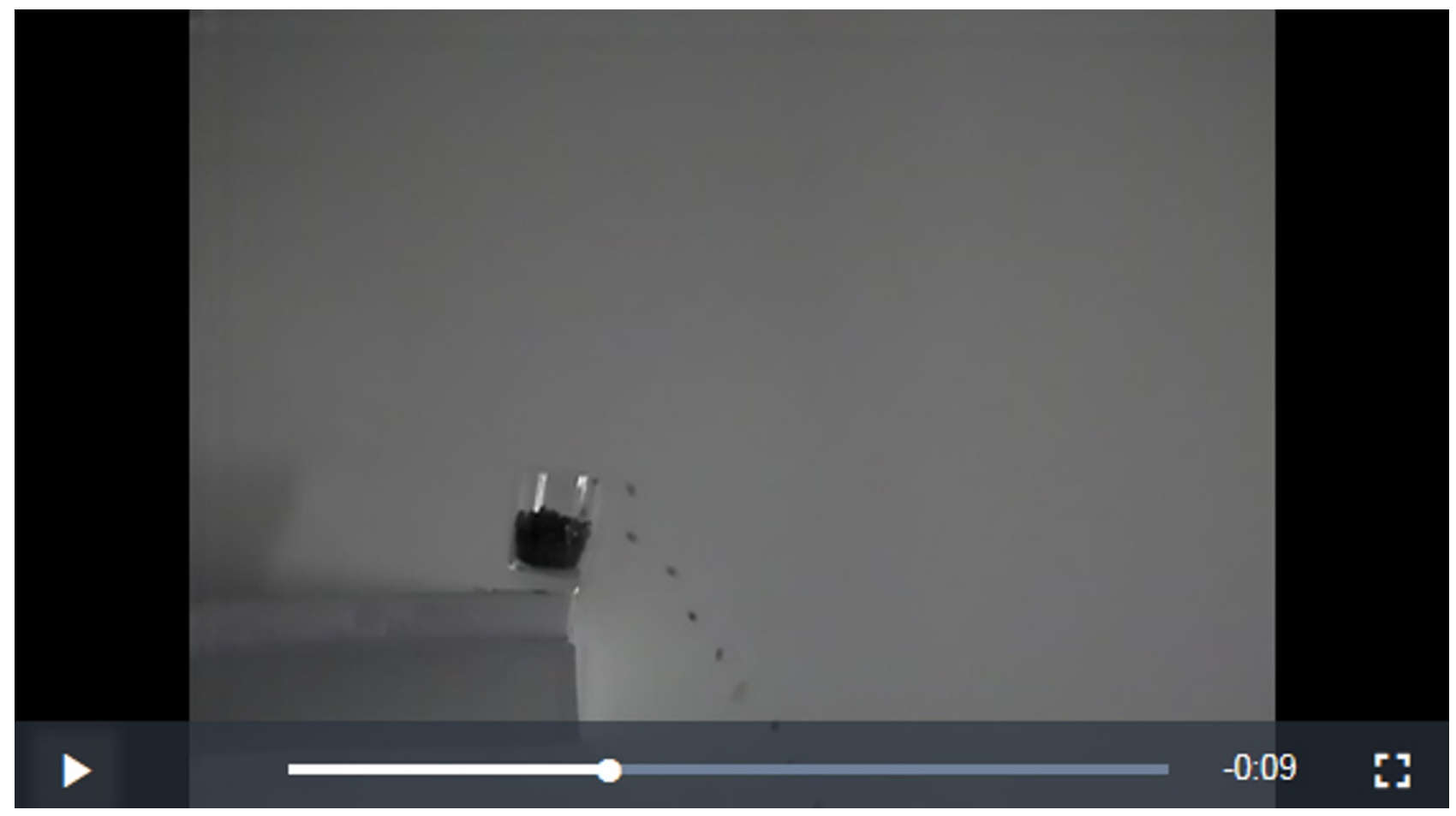

Nature I doi:10.1038/nature.2014.14523

\section{References}

1. Biggins, J. S. \& Warner, M. Proc. R. Soc. A 20130689 (2014).

2. Grewal, A. et al. Am. J. Phys. 79, 723-729 (2011). 